Erratum

\title{
Suzuki's Scaling Limit and the Theory of Statistical Fluctuations in Nuclear
} Fission

P. Grangé, H.C. Pauli, and H.A. Weidenmüller

Z. Physik A 296, 107-110 (1980)

The above article was erroneously placed in the section "Atoms". It should have appeared under "Nuclei".

P. Grangé

H.C. Pauli

H.A. Weidenmüller

Max-Planck-Institut

für Kernphysik

Postfach 103980

D-6900 Heidelberg 1

Federal Republic of Germany

and

Institut für Theoretische Physik

Universität Heidelberg

Philosophenweg 19

D-6900 Heidelberg 1

Federal Republic of Germany 